\title{
Archiv der Pharmazie
}

Dreier Antje und Haller Rolf

Dreier Antje und Haller Rolf

Abdelhamid Abdou $O$ and Riad Bahia Y.

Fini Adamo

Roda Aldo,

Bellini Anna Maria and Guarneri Mario

Eiden Fritz und Grusdt Ulrike

Knabe Joachim und Wahl Sebastian

Rehse Klaus, Sparfeldt Peter und Kehr Wolfgang

Wanner Klaus Th. und Kärtner Annerose

Lehmann Jochen und Gossen Axel

Reisch Johannes und Probst Wiltrud

Rehse Klaus,

Peetsch Rainer und Kehr Wolfgang

Glombitza Karl-Werner und Kurth Hermann

Jensen Jørgen A. and Pedersen Erik B.

Backe Werner
Inhalt

999

Gewinnung von 7-(3-Hydroxybutyl)-8chlortheophyllin und dem davon abgeleiteten Tetrahydro-oxazixanopurin in enantiomerenreiner Form

1004 Optisch aktive Tetrahydro-diazixanopurine

1010 Reaktionen mit 4-Brom-3-methyl-1-phenyl-2-pyrazolin-5-on: Neue anellierte Pyrazol-Derivate

1014 Dissoziationskonstanten von Hydroxy$5 \beta$-cholan-24-ammoniumionen

1020 4-Phenylthio-3-pyrrolcarbonsäureester

1032 2,6-Piperidindione, 4. Mitt.: Synthese der Racemate und der Enantiomere 3,3-disubstituierter 2,6-Piperidindione mit basischer Seitenkette

1042 Neuropsychotrope Aktivität dopaminanaloger Bicyclo[2,2,1]heptane

1050 Pd/C-katalysierte Doppelbindungsisomerisierung von $\mathrm{N}$-Acylallylaminen zu NAcylenaminen

1059 Lactone, 14. Mitt.: Synthese lactonverbrückter 1,1-Diarylethylamine

1065 Naturstoffchemie 110. Mitt.: Synthese des 1-Hydroxy-7-methoxyacridons aus Boenninghausenia albiflora REICHB. (Rutaceae); Darstellung einiger einfacher Hydroxy-, Methoxy- und Nitroacridone

1072 Rezeptorenaffinität ekliptisch fixierter Tryptamin- und Serotonin-Analoga

1083 Saponine aus Anagallis arvensis L. (Primulaceae)

1088 Phosphorentoxid in der organischen Synthese, 32. Mitt.: Eine neue Synthese des Quipazins und seines N-Methyl-Derivates

\section{Kurzmitteilung}

1093 Bestimmung von Flumequin in verschiedenen Produkten durch Hochdruckflüssigchromatographie

- Publikations-Sprache

\section{Contents}

Synthesis of 8-Chloro-7-(3-hydroxybutyl)theophylline and its Cyclization Product, a Tetrahydro-oxazixanopurine. as pure Enantiomers

Optically Active Tetrahydro-diazixanopurines

- Reactions with 4-Bromo-3-methyl-1phenyl-2-pyrazolin-5-one: New Fused Pyrazole Derivatives

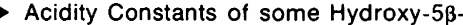
cholan-24-ammonium lons

4-(Phenylthio)-pyrrole-3-carboxylic Acid Esters

2,6-Piperidinediones, IV: Syntheses of the Racemates and the Enantiomers of 3,3-Disubstituted 2,6-Piperidinediones with Basic Side Chains

Neuropsychotropic Activity of Dopamin Analogous Bicyclo[2,2,1]heptanes

$\mathrm{Pd} / \mathrm{C}$-Catalysed Double Bond Isomerization of $\mathrm{N}$-Acyl-1,2,3,6-tetrahydropyridines to $\mathrm{N}$-Acyl-Enamines

Lactones, XIV: Synthesis of LactoneBridged 1,1-Diarylethanamines

Natural Product Chemistry. Part 110: Synthesis of 1-Hydroxy-7-methoxyacridone from Boenninghausenia albiflora REICHB. (Rutaceae). Preparation of Simple Hydroxy-, Methoxy- and Nitroacridones

Affinity to 5-HT Receptors of Tryptamin and Serotonin Analogues with Fixed Eclipsed Conformations

Saponins from Anagallis arvensis L. (Primulaceae)

- Phosphorus Pentoxide in Organic Synthesis XXXII: A New Synthesis of Quipazine and Its $N$-Methyl Derivatives

\section{Short Communication}

Determination of Flumequine in Various Preparations by HPLC

Language of Publication 

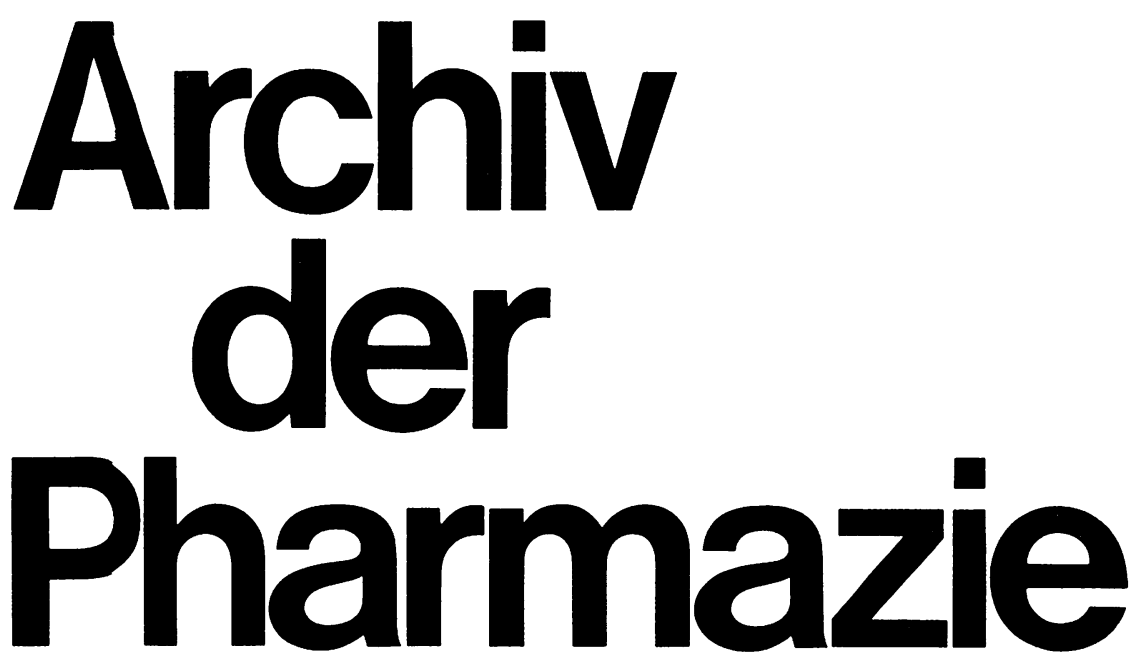

Zeitschrift für chemische, biologische und technologische Aspekte der Arzneimittelforschung, gegründet 1822
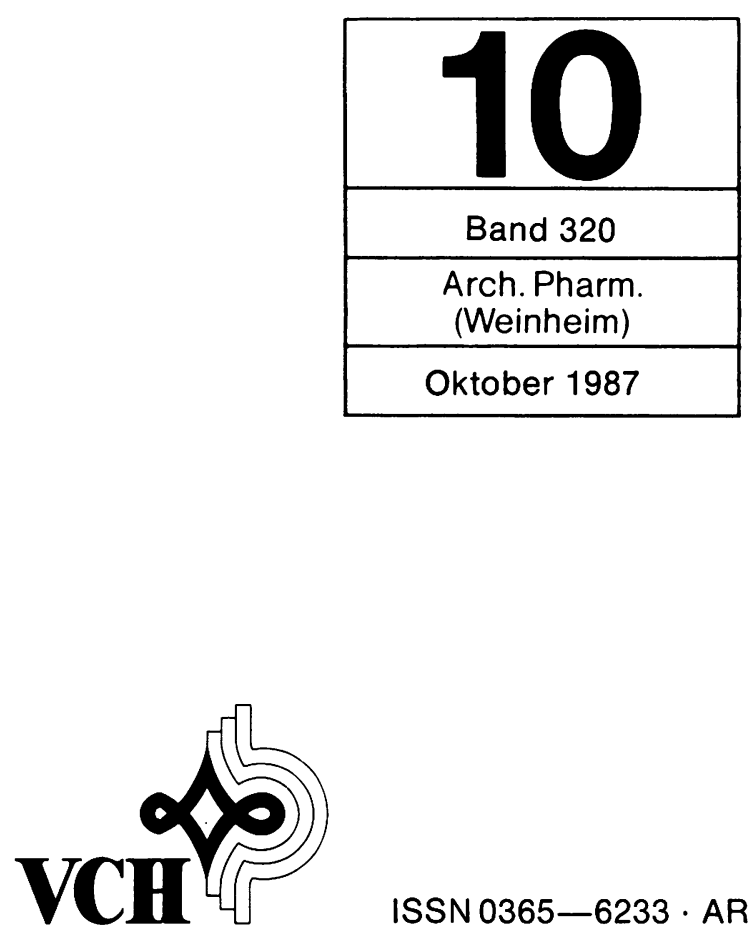


\section{Archiv der Pharmazie}

Herausgegeben von der Deutschen Pharmazeutischen Gesellschaft mit Unterstützung der Bundesvereinigung Deutscher Apothekerverbände - ABDA durch
W. Wiegrebe, Regensburg (Schriftleiter)
H. P. T. Ammon, Tübingen
F. Eiden, München
A. W. Frahm, Bonn
R. Hänsel, Berlin
K. Hartke, Marburg/L.
J. Knabe, Saarbrücken
H. König, Ludwigshafen
H. Möhrle, Düsseldorf

E. Mutschler, Frankfurt/M.

A. Nahrstedt, Münster

$H$. Oelschläger, Frankfurt/M.

H. J. Roth, Tübingen

H. Rupprecht, Regensburg

G. Seitz, Marburg

J. Thesing, Darmstadt

K. Thoma, München

SCHRIFTLEITUNG: Prof. Dr. W. Wiegrebe, Pharmazeutisches Institut der Universität Regensburg, Universitätsstr. 31, Postfach 397, D-8400 Regensburg.

Hinweis der Redaktion: Das Archiv der Pharmazie enthält Originalmitteilungen aus allen Gebieten der Pharmazeutischen Wissenschaften. Die Autoren werden gebeten, die Beiträge möglichst kurz abzufassen und dem Text eine straffe Zusammenfassung des Inhalts in deutscher und in englischer Sprache (mit englischem Titel) voranzustellen. Kostenlos veröffentlicht werden nur Manuskripte, die auf 8 Druckseiten untergebracht werden können. 8 Druckseiten entsprechen etwa 16 Schreibmaschinenseiten DIN A4 (30 Zeilen, 50 Anschläge, Rand 5 $\mathrm{cm}$ rechts) incl. Tabellen, Zeichnungen und Spektren. Für jede zusätzliche Druckseite werden dem Autor 60,$\mathrm{DM}$ in Rechnung gestellt. Kurzmitteilungen (incl. Formeln und Abbildungen insgesamt 4 Schreibmaschinenseiten), die keiner weiteren Publikation bedürfen, werden außerhalb der Reihe veröffentlicht. — Grundsätzlich werden nur Arbeiten angenommen, die bisher nicht veröffentlicht worden sind. Der Autor muß das alleinige Urheberrecht besitzen. Mit der Annahme des Manuskripts durch die Redaktion überträgt er der VCH Verlagsgesellschaft $\mathrm{mbH}$ das ausschließliche Nutzungsrecht, insbesondere das Recht der Vervielfältigung wie Fotokopie, Mikrofilm - oder mit irgendeinem anderen Verfahren - oder das Manuskript in eine von Maschinen, insbesondere von Datenverarbeitungsmaschinen verwendbare Sprache zu übergeben oder zu übersetzen (auch in andere Sprachen).

Eine Anweisung zur Abfassung von Manuskripten kann von der $\mathrm{VCH}$ Verlagsgesellschaft mbH bezogen werden. Es wird gebeten, die Korrekturen rasch zu erledigen. Verzögerte Korrekturen führen zur Zurückstellung des betreffenden Beitrages.

Zeitschriftenzitate sind abzukürzen nach den Angaben in „Bibliographic Guide for Editors \& Authors", herausgegeben von der American Chemical Society, 1974

Manuskripte sind zu senden an Prof. Dr. W. Wiegrebe, Universität, Postfach 397, D-8400 Regensburg.

Erscheinungsweise: monatlich. Jahresbezugspreis: 440,00 DM. Einzelheft 45,00 DM (1988: 470,00 DM bzw. 48,00 DM), zuzüglich Versandkosten. In diesen Preisen ist die gesetzliche Mehrwertsteuer enthalten. Die Mitglieder der Deutschen Pharmazeutischen Gesellschaft erhalten die Zeitschrift für 190,00 DM (1988: 256,00 DM), zuzüglich Versandkosten.

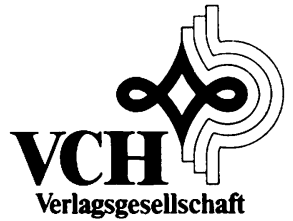

Verlag und Anzeigenabteilung: VCH Verlagsgesellschaft, Postfach 1260/ 1280, D-6940 Weinheim, Telefon: (06201) 602-0, Telex: 465516 vchwh d, Telefax (06201) 602328. Bestellungen richten Sie bitte an Ihre Fachbuchhandlung oder unmittelbar an den Verlag. Abbestellungen nur bis spätestens 3 Monate vor Ablauf des Kalenderjahres. Gerichtsstand und Erfüllungsort: D-6940 Weinheim. Lieferung erfolgt auf Rechnung und Gefahr des Empfängers. Anzeigen nach besonderem Tarif. Beilagen nach Vereinbarung.

For the USA and Canada: Published monthly by VCH Publishers, Inc., 303 N.W. 12th Avenue, Deerfield Beach FL 33442-1788; Telex 5101011104 VCHPUB; Telefax (305) 428-8201; Telephone (305) 428-5566 or (800) 422-8824. Second-class postage paid at Deerfield Beach FL 33441. Annual subscription price: US $\$ 244.00$ (1988: US $\$ 299.00$ ) including postage and handling charges. Printed in the Federal Republic of Germany. 
Arch. Pharm. (Weinheim) 320, 1050-1058 (1987)

\section{Pd/C-katalysierte Doppelbindungsisomerisierung von $\mathbf{N}$-Acylallylaminen zu $\mathbf{N}$-Acylenaminen}

Klaus Th. Wanner* und Annerose Kärtner

Institut für Pharmazie und Lebensmittelchemie der Universität München, Sopienstraße 10, 8000 München 2

Eingegangen am 21. Januar 1987

Die Synthesen optisch aktiver N-Acyl-1,2,3,6-tetrahydropyridine und deren Pd/C katalysierten Umlagerungen zu N-Acylenaminen werden beschrieben.

Pd/C-Catalysed Double Bond Isomerization of $\mathrm{N}$-Acyl-1,2,3,6-tetrahydropyridines to $\mathrm{N}$-AcylEnamines

The synthesis of optically active $N$-acyl-1,2,3,6-tetrahydropyridines and their Pd/C-catalysed rearrangements to $N$-acyl-enamines are described.

Enamide sind eine nützliche und vielseitige Substanzklasse, geeignet, mit Nucleophilen spezifisch in der $\alpha$ - und mit Elektrophilen spezifisch in der $\beta$-Position zu reagieren ${ }^{1)}$. Daran gemessen, werden Enamide verhältnismäßig selten verwandt; das mag z. T. an umständlichen und nicht allgemein anwendbaren Darstellungsmethoden liegen. 


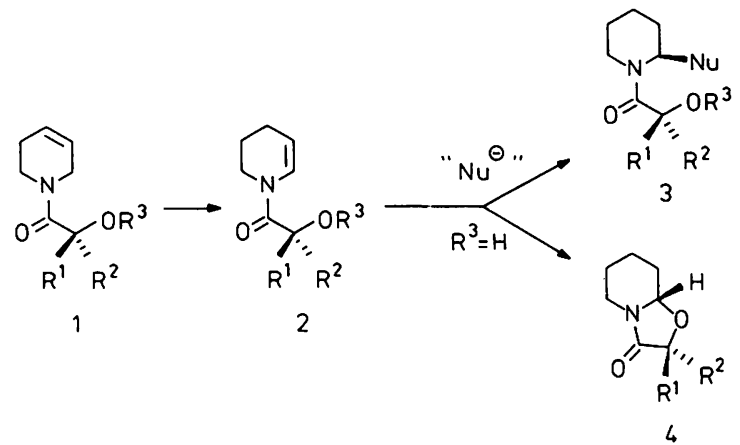

In Zusammenhang mit unseren Versuchen zur stereoselektiven Synthese pharmakologisch wirksamer Aminoalkohole ${ }^{2)}$ durch asymmetrische $\alpha$-Amidoalkylierung mit Kationenequivalenten $(2 \rightarrow 3)$ haben wir uns um einen effizienten Zugang zu Enamiden wie 2 bemüht. Darüber hinaus wollen wir mit diesen Enamiden Oxazolopyridine 4 gewinnen, deren antikonvulsive Eigenschaften dokumentiert sind ${ }^{3)}$.

Eine $\alpha$-Methoxylierung mit nachfolgender Methanolabspaltung ${ }^{4)}$ erschien hier nicht lohnenswert, da verzweigte Acylreste die Ausbeuten der anodischen Oxidation verringern sollen ${ }^{5}$. Ein weiteres Verfahren, die N-Acylierung von Iminen war nicht praktikabel, da das entsprechende Imin (1,2,3,4-Tetrahydropyridin) in freier Form nicht stabil ist $^{6)}$. Blieb als aussichtsreicher und direkter Weg die Doppelbindungsisomerisierung von $\mathbf{1} \rightarrow \mathbf{2}$. Solche Umlagerungen unter Verwendung spezieller, $z$. T. teurer und empfindlicher Eisen-, Rhodium- und Iridiumkatalysatoren sind beschrieben worden?).

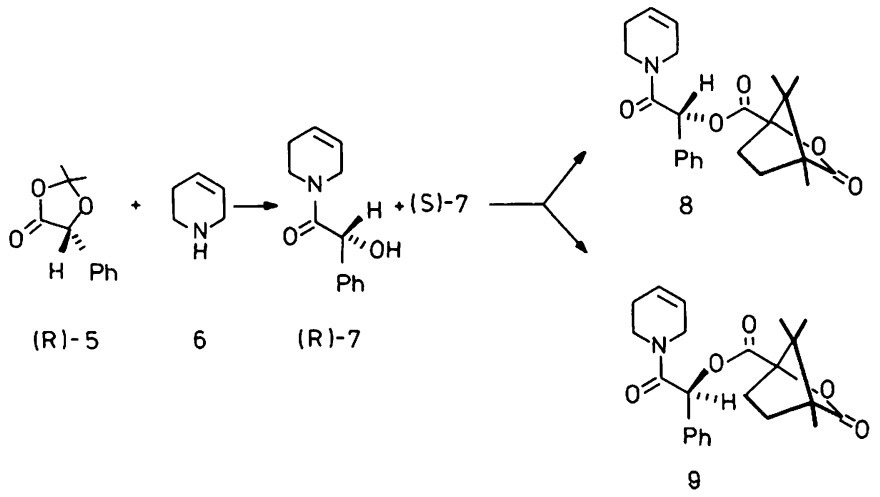

Um diesen Weg zu prüfen, haben wir einige $\mathrm{N}$-Acylderivate des Tetrahydropyridins (6) synthetisiert, die auch für eine asymmetrische $\alpha$-Amidoalkylierung geeignet sein sollten, z. B. das Mandelsäureamid (R)-7.

Dazu haben wir Mandelsäure als Dioxolan (R)-5 ${ }^{8}$ in Tetrahydrofuran mit Tetrahydropyridin 6 umgesetzt und dabei (R)-7/(S)-7 in $92.1 \%$ Ausbeute isoliert. Allerdings 
Tab. 1: Aminolyse des Dioxolans (R)-5 mit Tetrahydropyridin (6)

\begin{tabular}{cccccc}
\hline & \multicolumn{3}{c}{$(\mathrm{R})-7 /(\mathrm{S})-7$} & & \\
\cline { 2 - 4 } Nr. & {$[a]_{578}$} & {$[a]_{546}$} & Ausb. (\%) & Rkt.zeit (h) & Lösungsm. \\
\hline 1 & $0^{\circ}$ & $-0.5^{\circ}$ & 92.1 & 45 & THF \\
2 & $-31^{\circ}$ & $-35^{\circ}$ & 18.5 & 1.5 & $\mathrm{CH} \mathrm{OH}_{3}$ \\
3 & $-12^{\circ}$ & $-14^{\circ}$ & 82.9 & 44 & $\left(\mathrm{CH}_{3}\right)_{2} \mathrm{CHOH}$ \\
4 & $-37^{\circ}$ & $-40.5^{\circ}$ & 62.8 & 2.0 & $\mathrm{H}_{2} \mathrm{O}$ \\
5 & $-77.0^{\circ}$ & $-87.5^{\circ}$ & 14.5 & - & - \\
\hline
\end{tabular}

fiel die optische Aktivität so gering aus (Tab. $1 \mathrm{Nr}$. 1), daß wir ein zumindest teilweise racemisches Material vermuteten. Deshalb haben wir zunächst an racemischem 7 (dargestellt aus racemischem $5^{8}$ ) eine Methode zur Bestimmung der optischen Reinheit erarbeitet, die aus einer Veresterung von 7 mit (-)-Camphansäurechlorid (10) und liquidchromatographischer Analyse des Diastereomerengemisches 8, 9 besteht. Diese Methode, angewandt auf (R)-7/(S)-7 (Tab. 1 Nr. 1), bestätigte unsere Vermutung, daß es sich dabei um weitgehend racemisches Material handelt (ee $\leq 0.5 \%$ ).

Eine drastische Veränderung der optischen Drehung haben wir beim Wechsel des Reaktionsmediums beobachtet. In $\mathrm{MeOH}$ war die Reaktion innerhalb von $1.5 \mathrm{~h}$ vollständig abgelaufen, und das durch Zentrifugalchromatographie von mitentstandenem Mandelsäuremethylester getrennte (R)-7/(S)-7 drehte deutlich stärker (Tab. 1 Nr. 2). Anders in Isopropanol (Tab. 1 Nr. 3): Hier waren eine langsame Reaktion, hohe Ausbeuten und geringe optische Aktivität zu beobachten. Die besten Ergebnisse haben wir in einer heterogenen Mischung mit Wasser erzielt (Tab. 1 Nr. 4). Durch fraktionierte Kristallisation ließ sich die spezifische Drehung weiter steigern. Für ein Produkt mit den Drehwerten $[\alpha]_{546}=-83,0^{\circ}$ und $[\alpha]_{578}=-72,5^{\circ}$ haben wir mit unserer Methode (Camphansäurechlorid (10), HPLC-Analyse) die (R)-7/(S)-7 = 94/6 Zusammensetzung ermittelt. Nr. 5 der Tab. 1 zeigt ein typisches Ergebnis der fraktionierten Kristallisation. Durch Vergleich mit den Drehwerten des vorher beschriebenen Produkts bekannter Zusammensetzung haben wir auf eine optische Reinheit $>93 \%$ ((R)-7/(S)-7 $>96.5 / 3.5$ ) geschlossen.

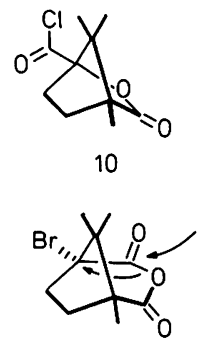

16
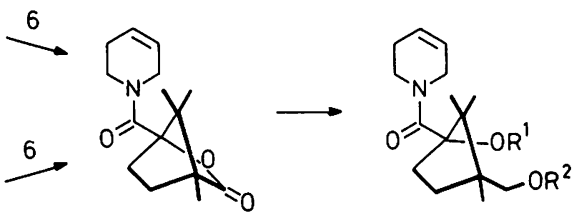

11

$$
\begin{aligned}
& \text { 1. } M H-12 \quad R^{1}, R^{2}=H \\
& \text { 2. } \mathrm{CH}_{3} \mathrm{I} \longrightarrow \mathrm{R}^{13}=\mathrm{H}, \mathrm{R}^{2}=\mathrm{CH}_{3} \\
& -60^{\circ} \rightarrow 0^{\circ} \longrightarrow 14 \quad R^{1}=\mathrm{CH}_{3}, R^{2}=\mathrm{H} \\
& 15 R^{\prime}=\mathrm{CH}_{3}, R^{2}=\mathrm{COPh}
\end{aligned}
$$


Tab. 2: Regioselektivtät der Alkylierung von 12

\begin{tabular}{llc}
\hline Nr. & MH & $13 / 14$ \\
\hline 1 & NaH & $51.4 / 48.6$ \\
2 & KH & $69.6 / 30.4$ \\
3 & KH + HMPA & $68.8 / 31.2$ \\
\hline
\end{tabular}

Mit einem konfigurationsstabilen Acylsubstituenten wie der Camphanoylgruppe ist die Gewinnung optisch reiner Amide kein Problem. 11 entstand bei der Reaktion von 6 mit (-)-Camphansäurechlorid (10) (Ausb. $77.8 \%$ ) und ebenso einfach und in guten Ausbeuten (82.2\%) bei der Reaktion von 6 mit Bromcamphersäureanhydrid") (16). Dieses Verfahren - der vermutete Reaktionsweg ist im Schema angedeutet - erlaubte es uns zwei Stufen zu überspringen, die sonst bei der über 16 verlaufenden Herstellung von 10 aus Camphersäure anfallen.

Weitere Amide mit chiralen und konfigurationsstabilen Acylgruppen haben wir wie folgt synthetisiert: Das Diol $\mathbf{1 2}$ war zugänglich durch reduktive Lactonspaltung mit $\mathrm{NaBH}_{4}$ in Diglyme (Ausb. 55-66 \%). In der Annahme, daß die primäre OH-Funktion von 12 acider als die tertiäre und 13 somit leicht zu gewinnen sei, haben wir 12 mit $\mathrm{NaH} / \mathrm{CH}_{3} \mathrm{~J}$ in THF umgesetzt. Das Produkt enthielt zwei Verbindungen in nahezu gleichen Teilen (Tab. 2 Nr. 1), deren NMR-Spektren fast gleich gut zu 13 wie 14 paßten. Für die Entscheidung in welcher Verbindung die primäre und in welcher die tertiäre OH-Gruppe methyliert ist, war das Benzoylderivat 15 nützlich. Das ${ }^{1} \mathrm{H}-\mathrm{NMR}$ Spektrum von 15 unterschied sich von der Vorstufe 14 vor allem in einer starken Tieffeldverschiebung der als AB-System auftretenden $\mathrm{CH}_{2} \mathrm{O}$-Gruppe (14: $\delta=3.25$; $3.59 \mathrm{ppm} ; 15: \delta=4.19 ; 4.59 \mathrm{ppm})$, die nur mit den Strukturen 14 und 15 vereinbar ist.

Als Ursache der mangelnden Regioselektivität ist ein Chelat des Natriumions zur benachbarten Carbonylgruppe zu vermuten, das die Bildung des tertiären Alkoxidions
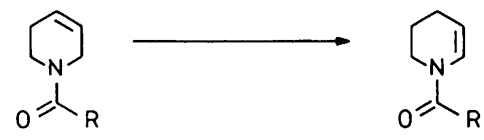

$7 \mathrm{R}=\mathrm{CHOH}-\mathrm{Ph}$
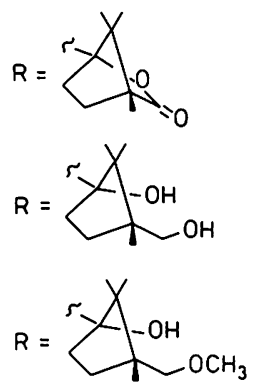
begünstigt. Um diese Wechselwirkung zu schwächen, haben wir $\mathrm{NaH}$ gegen $\mathrm{KH}$ ausgetauscht: Der Anteil des gewünschten Isomers 13 stieg tatsächlich auf $69.6 \%$ (Tab. 2 Nr. 2). Durch Zusatz von HMPA, das für seine dekomplexierende Wirkung bekannt ist, konnte allerdings keine zusätzliche Selektivitätssteigerung erzielt werden (Tab. 2 Nr. 3).

Die entscheidenden Untersuchungen zur Doppelbindungsisomerisierung nahmen wir an racemischem 7 vor. Mit Katalysatoren wie $\mathrm{RhCl}_{3} \operatorname{oder}\left(\mathrm{Ph}_{3} \mathrm{P}\right)_{3} \mathrm{RhCl}$ waren die Ergebnisse hinsichtlich Umsatz und Einheitlichkeit des Produkts unbefriedigend.

In $\mathrm{Pd} / \mathrm{C}$ fanden wir schließlich einen für die Isomerisierung der Allylamide besonders geeigneten Katalysator. Nach $6 \mathrm{~h}$ in THF bei $\sim 120^{\circ}$ war der Umsatz $>95 \%$ (NMR-spektroskopisch bestimmt); als Hauptprodukt lag 17 vor (HPLC-Analyse: $75 \%)$ begleitet von geringen Mengen stereoisomerer Oxazolopyridine $\left(4, \mathrm{R}^{1}=\mathrm{Ph}, \mathrm{R}^{2}\right.$ $=\mathrm{H}, 17 \% ; \mathbf{4}, \mathrm{R}^{1}=\mathrm{H}, \mathrm{R}^{2}=\mathrm{Ph}, 8 \%$ ). Deren Bildung - offensichtlich durch intramolekulare Cyclisierung - führten wir auf katalytisch wirksame Säurespuren zurück, die wir durch einen basischen Zusatz zu binden und inaktivieren versuchten. Unsere Annahme wurde bestätigt: Nach Zugabe von Triethylamin und Durchführung der Isomerisierung unter sonst gleichen Bedingungen waren die Oxazolopiperidine 4 selbst liquidchromatographisch nicht mehr nachweisbar, und die Ausbeuten an isoliertem 17 erreichten $80.5 \%{ }^{10}$.

Vergleichbar ließ sich 12 zu 19 (Ausb. $81.7 \%$ ), 13 zu 20 (Ausb. $80.1 \%$ ) und 11 zu 18 (Ausb. $90.7 \%$ ) isomerisieren. Beim letzten Beispiel war mangels einer zur Cyclisierung befähigten $\mathrm{OH}$-Gruppe in Nachbarschaft zur Carbonylgruppe kein $\mathrm{NEt}_{3}$-Zusatz $^{-}$ nötig, und - erwähnenswert - bei kleinen Ansätzen war bereits mit 1/50 Gewichtsprozent $\mathrm{Pd} / \mathrm{C}$ die Umsetzung quantitativ.

Auffallend bei den ${ }^{1} \mathrm{H}$-NMR-Spektren der Enamide ist die Tieffeldverschiebung von $\mathrm{H}-2(17 ; \delta=6.41 \mathrm{ppm})$, verglichen mit $\mathrm{H}-3(17 ; \delta=4.92 \mathrm{ppm})$. Außerdem ist ein zweiter, weniger intensiver Signalsatz zu beobachten, der durch gehinderte Rotation um die N-Acyl Bindung ${ }^{7)}$ zustande kommt.

Unseres Wissens stellt die hier beschriebene Gewinnung von Enamiden durch Doppelbindungsisomerisierung eine neue, bisher nicht bekannte Verwendung für Palladium auf Kohlenstoff dar ${ }^{11}$.

Herrn Prof. Dr. F. Eiden gilt unser besonderer Dank für die großzügige Förderung.

\section{Experimenteller Teil}

Schmp. (nicht korr.): Apparat nach Dr. Tottoli. - ${ }^{1}$ H-NMR-Spektren: T-60 und A-60 (Varian), WP 80, AM 250 und AM 360 (Bruker); $\delta$-Skala (ppm), TMS int. Stand. - MS: CH 7 (Varian). - IR-Spektren: Acculab 6 (Beckman); Flüssigkeiten als Film. Feststoffe als $\mathrm{KBr}$-Preßlinge. - Optische Drehungen: Lichtelektrisches Polarimeter Zeiss, 0.5-dm-Rohr. - CHN-Analysen: CHN-Rapid (Heraeus). - SC: Flash Chromatographie ${ }^{12)}$. - Zentrifugalchromatographie: Chromatotron (Harrison Research), Si 60. Schutzgas: $\mathrm{N}_{2}$. - HPLC-Apparatur: Chromatographiepumpe 6000A, Brechungsindexdetektor R 401, UV-Detektor 440; $254 \mathrm{~nm}$ (Waters); LiChroCart. LiChrosorb Si60 HPLC-Kartusche ( $250 \mathrm{~mm} \times 4 \mathrm{~mm}$ i. D.) und LiChroCart, LiChrosorb Si $60 \mathrm{Kartuschenvorsäule} \mathrm{(25} \mathrm{mm} \times 4 \mathrm{~mm}$ i. D., Merck). 


\section{(R,S)-1-(1,2,3,6-Tetrahydro-1-pyridyl)-2-hydroxy-2-phenylethanon (7)}

$0.96 \mathrm{~g}(5 \mathrm{mmol}) 5^{8)}$ und $0.83 \mathrm{~g}(10 \mathrm{mmol}) 6 \mathrm{in} 2.5 \mathrm{ml}$ absol. THF wurden $45 \mathrm{~h}$ bei Raumtemp. gerührt: Nach Einengen i. Vak. wurde mit Ethylacetat versetzt, mit $0.5 \mathrm{~N} \mathrm{HCl}(3 \times), 0.5 \mathrm{~N} \mathrm{NaOH}(2 \times)$ und Wasset $(2 \times)$ geschüttelt, getrocknet $\left(\mathrm{MgSO}_{4}\right)$ und das Lösungsmittel i. Vak. abgedampft. Farblose Kristalle, Schmp. 71-72.5 (Diisopropylether), Ausb. $0.9 \mathrm{~g} \mathrm{(82.8 \% ).} \mathrm{-} \mathrm{C}_{13} \mathrm{H}_{15} \mathrm{NO}_{2}$ (217.3). Ber. C $71.9 \mathrm{H} 6.96$ N 6.5 Gef. C 71.8 H 6.79 N 6.5 Mol.-Masse 217 (ms). - IR: 3500-3100; $1635 \mathrm{~cm}^{-1}$. - ${ }^{1} \mathrm{H}-\mathrm{NMR}$ $\left(\mathrm{CDCl}_{3}\right): 1.30-2.33 \mathrm{ppm}\left(\mathrm{m}, 2 \mathrm{H},=\mathrm{C}-\mathrm{CH}_{2}-\right), 3.12-4.28\left(\mathrm{~m}, 4 \mathrm{H},-\mathrm{CH}_{2} \mathrm{NCH}_{2}-\right), 4.70-4.92(\mathrm{~m}, 1 \mathrm{H}, \mathrm{OH}$, $\mathrm{D}_{2} \mathrm{O}$-Austausch), 5.25 (d, J = 6.0 Hz, 1H, H-C-O), 5.40-6.00 (m, 2H, $\left.-\mathrm{CH}=\mathrm{CH}-\right), 7.35$ (zentr. $\mathrm{m}, 5 \mathrm{H}$, $-\mathrm{C}_{6} \mathrm{H}_{5}$ ).

\section{(R)-1-(1,2,3,6-Tetrahydro-1-pyridyl)-2-hydroxy-2-phenylethanon ((R)-7)}

In einer Mischung aus $20 \mathrm{ml}$ Wasser und $5.0 \mathrm{~g}$ ( $60 \mathrm{mmol}) 6$ wurden bei Raumtemp. $3.84 \mathrm{~g}(20 \mathrm{mmol})$ fein gepulvertes (R)-5 ${ }^{8)}$ suspendiert. Nach $2 \mathrm{~h}$ wurde das vorübergehend homogen gewordene Reaktionsgemisch abgesaugt (Rückstand weitgehend racemisches Produkt), mit $50 \mathrm{ml} 1 \mathrm{~N} \mathrm{HCl}$ und Ethylacetat versetzt und 0.5 h bei Raumtemp. gerührt. Die org. Phase wurde mit $0.1 \mathrm{~N} \mathrm{HCl}(2 \times)$, gesättigter $\mathrm{NaHCO}_{3}$ Lösung $(2 \times)$ und $\mathrm{NaCl}$-Lösung $(2 \times)$ geschüttelt, getrocknet $\left(\mathrm{Na}_{2} \mathrm{SO}_{4}\right)$, eingeengt und der Rückstand $(1.58 \mathrm{~g})$ aus Diethylether fraktioniert kristallisiert. Farblose Kristalle, Schmp. $60-63^{\circ},[\alpha]_{546}=-87.5^{\circ}$; $|\alpha|_{578}=-77.0^{\circ}\left(\mathrm{c}=2.0, \mathrm{CH}_{3} \mathrm{OH}\right)$, Ausb. $0.63 \mathrm{~g}(14.5 \%) .-\mathrm{C}_{13} \mathrm{H}_{15} \mathrm{NO}_{2}$ (217.3). Ber. C $71.9 \mathrm{H} 6.96$ N 6.5 Gef. C 71.7 H 6.95 N 6.5. - Mol.-Masse, IR, ' $\mathrm{H}$-NMR wie bei 7 beschrieben.

\section{Diastereomere Ester $(\mathbf{8}, 9)$ der (-)-Camphansäure}

a) Eine Mischung aus $43 \mathrm{mg}(0.2 \mathrm{mmol}) 7,65 \mathrm{mg}(0.3 \mathrm{mmol}) 10$ und $23.7 \mathrm{mg}(0.3 \mathrm{mmol})$ Pyridin in $0.5 \mathrm{ml}$ absol. $\mathrm{CH}_{2} \mathrm{Cl}_{2}$ wurde $2 \mathrm{~h}$ bei Raumtemp. gerührt und nach Zusatz von $0.2 \mathrm{ml} \mathrm{H}_{2} \mathrm{O}$ weitere $0.5 \mathrm{~h}$. Es wurde mit Ethylacetat verdünnt, mit $0.5 \mathrm{~N} \mathrm{HCl}(3 \mathrm{x})$, mit gesättigter $\mathrm{NaHCO}_{3}$-Lösung $(2 \times)$ und mit $\mathrm{H}_{2} \mathrm{O}(2 \times)$ geschüttelt, getrocknet $\left(\mathrm{Na}_{2} \mathrm{SO}_{4}\right)$ und eingeengt. Farblose Kristalle, Schmp. 150-157 . $\mathrm{C}_{23} \mathrm{H}_{27} \mathrm{NO}_{5}$ (397.5), Ber. C 69.5 H 6.85 N 3.5 Gef. C 69.6 H 6.93 N 3.3 Mol.-Masse 397 (ms). - IR: $1790 ; 1760 ; 1645 \mathrm{~cm}^{-1}$. - 'H-NMR $\left(\mathrm{CDCl}_{3}, 80 \mathrm{MHz}\right): 1.04,1.09,1.10,1.14,1.25\left(5 \mathrm{~s}, 9 \mathrm{H}, \mathrm{C}\left(\mathrm{CH}_{3}\right)_{2}\right.$, $\left.\mathrm{CH}_{3}\right), 1.25-2.80(\mathrm{~m}, 6 \mathrm{H}), 3.25-4.25\left(\mathrm{~m}, 4 \mathrm{H},-\mathrm{CH}_{2} \mathrm{NCH}_{2}-\right), 5.25-5.95(\mathrm{~m}, 2 \mathrm{H},-\mathrm{CH}=\mathrm{CH}-), 6.38(\mathrm{~s}$, breit,H-C-O), 7.45 (zentr. m, $5 \mathrm{H},-\mathrm{C}_{6} \mathrm{H}_{5}$ ). - HPLC-Analyse: Hexan $/$ Ethylacetat $=60: 40,1.50 \mathrm{ml} / \mathrm{min}$. 9: $4.36 \mathrm{~min}, 50.4 \% ; 8: 5.00 \mathrm{~min}, 49.6 \%$.

b) Analoge Umsetzung wie bei a) von (R)-7 $\left([\alpha]_{546}=-83.0^{\circ},[\alpha]_{578}=-72.5^{\circ}, \mathrm{c}=2.0, \mathrm{CH}_{3} \mathrm{OH}\right)$. HPLCAnalyse: 9: $6.0 \% ; 8: 94.0 \%$.

\section{(IS, 4R)-1-(1,2,3,6-Tetrahydro-1-pyridylcarbonyl)-4,7,7-trimethyl-2-oxabicyclol 2.2.1 Jheptan-3-on (11)}

a) $1.05 \mathrm{~g}(4.8 \mathrm{mmol}) 10$ in $10 \mathrm{ml}$ absol. $\mathrm{CH}_{2} \mathrm{Cl}_{2}$ wurden unter Eiskühlung mit $\left.0.53 \mathrm{~g} \mathrm{(5.3} \mathrm{mmol}\right) \mathrm{NEt}_{3}$ versetzt, gefolgt von $0.44 \mathrm{~g}(5.3 \mathrm{mmol}) 6$. Es wurde $1 \mathrm{~h}$ bei $0^{\circ}$ und $4 \mathrm{~h}$ bei Raumtemp. gerührt, mit $\mathrm{CH}_{2} \mathrm{Cl}_{2}$ verdünnt, abwechselnd mit $\mathrm{H}_{2} \mathrm{O}$ und mit $1 \mathrm{~N} \mathrm{HCl}$ geschüttelt, getrocknet $\left(\mathrm{Na}_{2} \mathrm{SO}_{4}\right)$ eingeengt und aus Diisopropylether umkristallisiert. Farblose Kristalle, Schmp. 85-86 $,[\alpha]_{546}=40.0^{\circ},[\alpha]_{578}=-33.0^{\circ}(\mathrm{c}=$ 1.0, $\left.\mathrm{CH}_{3} \mathrm{OH}\right)$, Ausb. $1.14 \mathrm{~g}\left(77.8 \%\right.$. $-\mathrm{C}_{15} \mathrm{H}_{21} \mathrm{NO}_{3}$ (263.3) Ber. C $68.4 \mathrm{H} 8.04$ N 5.32 Gef. C 68.5 H $7.82 \mathrm{~N} 5.42$ Mol.-Masse 263 (ms). - IR: 3040; 1780; $1630 \mathrm{~cm}^{-1}$. - ' $\mathrm{H}-\mathrm{NMR}\left(\mathrm{CDCl}_{3}\right): 1.02,1.12,1.20$ $\left(3 \mathrm{~s}, 9 \mathrm{H}, \mathrm{C}\left(\mathrm{CH}_{3}\right)_{2}, \mathrm{CH}_{3}\right), 1.53-2.70(\mathrm{~m}, 10 \mathrm{H}), 3.43-4.50\left(\mathrm{~m}, 4 \mathrm{H},-\mathrm{CH}_{2} \mathrm{NCH}_{2}-\right), 5.50-6.08(\mathrm{~m}, 2 \mathrm{H}$, $-\mathrm{CH}=\mathrm{CH}-)$.

b) $\left.26.1 \mathrm{~g}(100 \mathrm{mmol}) 16^{9}\right)$ in $200 \mathrm{ml}$ absol. THF wurden unter Eiskühlung mit $12.45 \mathrm{~g}$ (150 mmol) 6 versetzt, gefolgt von $15.2 \mathrm{~g} \mathrm{(150} \mathrm{mmol)} \mathrm{NEt}_{3}$. Das Gemisch wurde dann $24 \mathrm{~h}$ ohne das Eisbad zu erneuern gerührt und i. Vak. vom Lösungsmittel befreit. Es wurde mit Ethylacetat versetzt, abwechselnd mit $\mathrm{H}_{2} \mathrm{O}$ und $1 \mathrm{~N} \mathrm{HCl}$ geschüttelt, getrocknet $\left(\mathrm{Na}_{2} \mathrm{SO}_{4}\right)$, i. Vak. eingeengt und aus Diisopropylether umkristallisiert. Ausb. 21.65 g (82.2 \%). 
(1,2,3,6-Tetrahydro-1-pyridyl)-I(1S, 3R)-1-hydroxy-3-hydroxymethyl-2,2,3-trimethylcyclopentyl/keton (12)

Eine Mischung aus 15,8 g (60 mmol) $11,3.4 \mathrm{~g}(90 \mathrm{mmol})$ fein gepulvertem $\mathrm{NaBH}_{4}$ und $300 \mathrm{ml}$ Diethylenglykoldimethylether wurde solange bei $120^{\circ}$ (Badtemp.) gerührt, bis $11 \mathrm{dc}$ nicht mehr nachzuweisen war (ca. 4 d). Es wurde $\mathrm{H}_{2} \mathrm{O}(150 \mathrm{ml})$ zugesetzt, $0.5 \mathrm{~h}$ bei Raumtemp. gerührt und mehrfach mit $\mathrm{CH}_{2} \mathrm{Cl}_{2}$ extrahiert. Die Extrakte wurden mit $\mathrm{H}_{2} \mathrm{O}$ geschüttelt. getrocknet $\left(\mathrm{Na}_{2} \mathrm{SO}_{4}\right)$ und i. Vak. weitgehend eingeengt, worauf sich 12 (z. T. geringfügig mit 19 kontaminiert) in Form farbloser Kristalle ausschied. Schmp. $118^{\circ} .[\alpha]_{546}=+80.2^{\circ},[\alpha]_{578}=+70.3^{\circ}\left(\mathrm{c}=2.02, \mathrm{CH}_{3} \mathrm{OH}\right)$, Ausb. 8.8-10.6 g (55-66 \%). $-\mathrm{C}_{15} \mathrm{H}_{25} \mathrm{NO}_{3}$ (267.4) Ber. C 67.4 H 9.42 N 5.2 Gef. C 67.6 H 9.29 N 5.1 Mol.-Masse 267 (ms). - IR: 3500-2700; $1630 ; 1595 \mathrm{~cm}^{-1}$. - ' $\mathrm{H}-\mathrm{NMR}\left(\mathrm{CDCl}_{3}\right): 0.83$ (s. 6H. $\left.\mathrm{CH}_{3}\right), 1.08\left(\mathrm{~s}, 3 \mathrm{H}, \mathrm{CH}_{3}\right), 1.30-2.40(\mathrm{~m}, 6 \mathrm{H})$, 2.62-4.90 (m, 4H, $-\mathrm{CH}_{2} \mathrm{NCH}_{2}$ ) $), 3.24,3.55$ (AB-System. $\mathrm{J}=10 \mathrm{~Hz}, 2 \mathrm{H},-\mathrm{CH}_{2} \mathrm{O}-$ ), 5.30-6.10 (m, 4H. $2 \mathrm{H}$ $\mathrm{D}_{2} \mathrm{O}$-Austausch, $-\mathrm{CH}=\mathrm{CH}-, \mathrm{CO} \underline{\mathrm{H}},-\mathrm{CH}_{2} \mathrm{O}-$ $)$.

(1,2,3,6-Tetrahydro-1-pyridyl)-I(1S, 3R)-1-hydroxy-3-methoxymethyl-2,2,3-trimethylcyclopentyl/keton (13) und

(1,2,3,6-Tetrahydro-1-pyridyl)I(IS, 3R)-3-hydroxymethyl-1-methoxy-2,2,3-trimethylcyclopentyl/keton (14)

$0.65 \mathrm{~g}(16.2 \mathrm{mmol}) \mathrm{KH}$ wurden in $7.5 \mathrm{ml}$ absol. THF suspendiert und unter Eiskühlung tropfenweise mit $3.94 \mathrm{~g}(14.7 \mathrm{mmol}) 12 \mathrm{in} 22.5 \mathrm{ml}$ absol. THF versetzt. Nach $1.5 \mathrm{~h}$ wurde auf $-60^{\circ}$ abgekühlt und mit $3.12 \mathrm{~g}(21.05 \mathrm{mmol}) \mathrm{CH}_{3} \mathrm{I}$ versetzt. Das Gemisch wurde langsam auf $-40^{\circ}$ und nach $3 \mathrm{~h}$ bei dieser Temp. auf Raumtemp. erwärmt. Nach Zugabe von $12 \mathrm{ml} \mathrm{10proz.} \mathrm{NH}_{4} \mathrm{Cl}$-Lösung wurde mit Ethylacetat verdünnt, festes $\mathrm{Na}_{2} \mathrm{~S}_{2} \mathrm{O}_{3}$ zugesetzt mit gesättigter $\mathrm{NaCl}$-Lösung geschüttelt, getrocknet $\left(\mathrm{Na}_{2} \mathrm{SO}_{4}\right)$ und i. Vak. eingeengt. Anschließend wurde der Rückstand sc in 13 und 14 getrennt (Hexan/Ethylacetat $=60 /$ 40; 13 vor 14). 13: Farblose Kristalle; Schmp. $70-73^{\circ} .[\alpha]_{546}=+73.1,[\alpha]_{578}=+64.4^{\circ}(\mathrm{c}=2.07$. $\mathrm{CH}_{3} \mathrm{OH}$ ), Ausb. $2.5 \mathrm{~g}(60.4 \%)$. $-\mathrm{C}_{16} \mathrm{H}_{27} \mathrm{NO}_{3}$ (281.4) Ber. C $68.3 \mathrm{H} 9.67 \mathrm{~N} 5.0$ Gef. C $68.0 \mathrm{H} 9.58$ N 4.9 Mol.-Masse 281 (ms). - IR: 3500-3200; $1605: 1110 \mathrm{~cm}^{-1}$. - ${ }^{1} \mathrm{H}-\mathrm{NMR}\left(\mathrm{CDCl}_{3}\right): 0.86\left(\mathrm{~s}, 6 \mathrm{H}, \mathrm{CH}_{3}\right)$, $1.03\left(\mathrm{~s}, 3 \mathrm{H}, \mathrm{CH}_{3}\right), 1.26-2.23(\mathrm{~m}, 6 \mathrm{H}), 3.06,3.33$ (AB-System, J $\left.=9.5 \mathrm{~Hz}, 2 \mathrm{H},-\mathrm{CH}_{2} \mathrm{O}-\right), 3.43(\mathrm{~s} .3 \mathrm{H}$, $\left.\mathrm{OCH}_{3}\right), 2.8-4.9\left(\mathrm{~m}, 4 \mathrm{H},-\mathrm{CH}_{2} \mathrm{NCH}_{2}-\right), 5.50\left(\mathrm{~s}, 1 \mathrm{H}, \mathrm{D}_{2} \mathrm{O}\right.$-Austausch, $\left.\mathrm{OH}\right), 5.53-6.09(\mathrm{~m}, 2 \mathrm{H},-\mathrm{CH}=\mathrm{CH}-)_{\text {. }}$

14: Farblose Kristalle, Schmp. 65-70 N 5.0 Gef. C 68.6 H 9.55 N 4.8 Mol.-Masse 281 (ms). - IR: 3600-3100; 1620; $1425 \mathrm{~cm}^{-1}$. - 'H-NMR $\left(\mathrm{CDCl}_{3}\right): 0.90\left(\mathrm{~s}, 6 \mathrm{H}, \mathrm{CH}_{3}\right), 1.07,1.12\left(2 \mathrm{~s}, 3 \mathrm{H}, \mathrm{CH}_{3}\right.$, Aufspaltung wegen Rotationsisomerer $), 1.25-2.48$ $(\mathrm{m}, 6 \mathrm{H}), 3.30$ (s, breit, $\left.3 \mathrm{H}, \mathrm{OCH}_{3}\right), 3.25$ und $3.59\left(\mathrm{AB}-\mathrm{System}, 2 \mathrm{H},-\mathrm{CH}_{2} \mathrm{O}-\right), 2.48-4.70(\mathrm{~m}, 4 \mathrm{H}$, $\left.-\mathrm{CH}_{2} \mathrm{NCH}_{2}-\right), 5.50-6.10$ (m, 2H, $-\mathrm{CH}=\mathrm{CH}$-). - HPLC-Analyse: Hexan/Ethylacetat $=60 / 40,1.50 \mathrm{ml} /$ min. Zur Probenaufbereitung wurde das Reaktionsgemisch nach dem es Raumtemp. erreicht hatte i. Vak. von THF und $\mathrm{CH}_{3} \mathrm{I}$ befreit und der Rückstand im HPLC-Laufmittel suspendiert. Anschließend wurde zentrifugiert und die klare Lösung direkt gemessen. 13: $4.27 \mathrm{~min}, 69.6 \%$; 14: $9.10 \mathrm{~min} .30 .4 \%$.

$I(1 R, 3 S)-3-(1,2,3,6-T e t r a h y d r o-1-p y r i d y l c a r b o n y l)-3-m e t h o x y-1,2,2-t r i m e t h y l-c y c l o p e n t y l m e t h y l /-$ benzoat (15)

$56.3 \mathrm{mg}(0.2 \mathrm{mml}) 14,36.5 \mathrm{mg}(0.26 \mathrm{mmol})$ Benzoylchlorid, $26.3 \mathrm{mg}(0.26 \mathrm{mmol}) \mathrm{NEt}_{3}$ und $0.4 \mathrm{ml}$ $\mathrm{CH}_{2} \mathrm{Cl}_{2}$ wurden $24 \mathrm{~h}$ auf $40^{\circ}$ erwärmt. Dann wurde mit $\mathrm{CH}_{2} \mathrm{Cl}_{2}$ verdünnt, mit $\mathrm{H}_{2} \mathrm{O}$ geschüttelt, getrocknet $\left(\mathrm{Na}_{2} \mathrm{SO}_{4}\right)$ eingeengt und zentrifugalchromatographisch gereinigt (Hexan/Ethylacetat $\left.=75: 25\right)$. Mol. Masse 385 (ms). - IR: 3040; 1720; $1625 \mathrm{~cm}^{-1}$. - ' $\mathrm{H}-\mathrm{NMR}\left(\mathrm{CDCl}_{3}, 80 \mathrm{MHz}\right): 0.86\left(\mathrm{~s}, 3 \mathrm{H}, \mathrm{CH}_{3}\right), 1.06(\mathrm{~s}$. $\left.6 \mathrm{H}, \mathrm{CH}_{3}\right), 1.33-2.39(\mathrm{~m}, 6 \mathrm{H}), 3.21,3.25\left(2 \mathrm{~s}, 3 \mathrm{H}, \mathrm{OCH}_{3}\right.$, Aufspaltung wegen Rotationsisomerer $), 4.19$. 4.51 (AB-System, J = 11,0 Hz, 2H, - $\mathrm{CH}_{2} \mathrm{O}-$ ). 2.63-3.09. 3.30-4.75 (2m, 4H, $-\mathrm{CH}_{2} \mathrm{NCH}_{2}$ ), 5.49-6.05 (m, $2 \mathrm{H},-\mathrm{CH}=\mathrm{CH}-), 7.31-7.71(\mathrm{~m}, 3 \mathrm{H}$, aromat.), 7.92-8.19 (m, 2H, aromat.). 


\section{Katalytische Doppelbindungsisomerisierung, Allgemeine Vorschrift}

Eine 1.0 molare Lösung des Amids in THF/NEt ${ }_{3}(8 / 2)$ wurde mit $1 / 20$ Gewichtsteil Pd/C (10\% Pd) unter Rühren in einem Druckrohr auf $115-120^{\circ}$ (Badtemp.) erhitzt. Nach entspr. Reaktionszeit wurde filtriert und aufgearbeitet.

\section{(R,S)-1-(1,2,3,4-Tetrahydro-1-pyridyl)-2-hydroxy-2-phenylethanon (17)}

$0.22 \mathrm{~g} \mathrm{(1} \mathrm{mmol)} \mathrm{7,6} \mathrm{h.} \mathrm{-} \mathrm{Das} \mathrm{Filtrat} \mathrm{wurde} \mathrm{i.} \mathrm{Vak.} \mathrm{eingeengt} \mathrm{und} \mathrm{der} \mathrm{Rückstand} \mathrm{zentrifugalchromatogra-}$ phisch $\left(\mathrm{CH}_{2} \mathrm{Cl}_{2} /\right.$ EtOAc $\left.=93 / 7\right)$ gereinigt. Farblose Kristalle; Schmp. 95-97.5 (Hexan/Ethylacetat), Ausb. $0.177 \mathrm{~g}\left(80.5 \%\right.$ ). $\mathrm{C}_{13} \mathrm{H}_{15} \mathrm{NO}_{2}$ (217.3) Ber. C $71.9 \mathrm{H} 6.96 \mathrm{~N} 6.5$ Gef. C $71.9 \mathrm{H} 6.95$ N 6.5 Mol.Masse 217 (ms). - IR: 3500-3200; 1650; $1420 \mathrm{~cm}^{-1}$. - 'H-NMR (CDCl, $\left.360 \mathrm{MHz}\right): 1.53-1.92(\mathrm{~m}, 2 \mathrm{H}$,

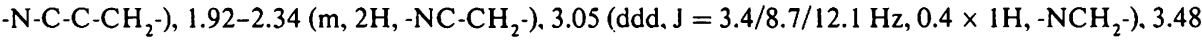
$\left(\mathrm{ddd}, \mathrm{J}=3.5 / 7.2 / 12.1 \mathrm{~Hz}, 0.4 \times 1 \mathrm{H},-\mathrm{NCH}_{2}-\right.$ ). 3.72 (ddd. $\mathrm{J}=3.9 / 7.6 / 12.9 \mathrm{~Hz}, 0.6 \times 1 \mathrm{H},-\mathrm{NCH}_{2}-$ ). 3.85 (ddd, J = 3.9/7.6/12.9 Hz, $\left.0.6 \times 1 \mathrm{H},-\mathrm{NCH}_{2}-\right), 4.59\left(\mathrm{~d} . \mathrm{J}=6.6 \mathrm{~Hz}, 0.6 \times 1 \mathrm{H}, \mathrm{D}_{2} \mathrm{O}\right.$-Austausch, $\left.\mathrm{OH}\right), 4.68$ $\left(\mathrm{d}, \mathrm{J}=6,4 \mathrm{~Hz}, 0.4 \times 1 \mathrm{H}, \mathrm{D}_{2} \mathrm{O}\right.$-Austausch, $\left.\mathrm{OH}\right), 4.92(\mathrm{dt} . \mathrm{J}=3.9 / 8.4 \mathrm{~Hz}, 0.6 \times 1 \mathrm{H},-\mathrm{NC}=\mathrm{CH}$ ), 5.18 (dt. $\mathrm{J}=3.9 / 8.4 \mathrm{~Hz}, 0.4 \times 1 \mathrm{H},-\mathrm{NC}=\mathrm{CH}-), 5.27(\mathrm{~d}, \mathrm{~J}=6.4 \mathrm{~Hz}, 0.4 \times 1 \mathrm{H}, \mathrm{PhCH}-), 5.30(\mathrm{~d}, \mathrm{~J}=6.6 \mathrm{~Hz}, 0.6 \times$ $1 \mathrm{H}, \mathrm{PhCH}-), 6.41(\mathrm{dt}, \mathrm{J}=1.9 / 8.4 \mathrm{~Hz}, 0.6 \times 1 \mathrm{H},-\mathrm{NCH}=\mathrm{C}-), 7.21(\mathrm{dt}, \mathrm{J}=2.0 / 8.4 \mathrm{~Hz}, 0.4 \times 1 \mathrm{H}$, $\mathrm{NCH}=\mathrm{C}-)$, 7.25-7.43 (m, 5H, $\left.-\mathrm{C}_{6} \mathrm{H}_{5}\right)$. - Rotamerenverhältnis $6 / 4$.

\section{(IS, 4R)-1-(1,2,3,4-Tetrahydro-1-pyridyl-carbonyl)-4,7,7-trimethyl-2-oxabicyclo/2.2.1/heptan-3-on (18)}

Abweichend von der allgemeinen Vorschrift:

a) $1.317 \mathrm{~g} \mathrm{(5} \mathrm{mmol)} 11,26 \mathrm{mg} \mathrm{Pd} / \mathrm{C}, 10 \mathrm{ml}$ THF, $3 \mathrm{~h}, 110^{\circ}$. - Das Filtrat wurde i. Vak. eingeengt und der Rückstand sc gereinigt (Hexan/Ethylacetat $=80 / 20)$. Farblose Kristalle; Schmp. $125-129^{\circ}(\mathrm{Hexan} /$ Ethylacetat), $[\alpha]_{546}=+4.0^{\circ},[\alpha]_{578}=+3.0^{\circ}\left(\mathrm{c}=1.0 . \mathrm{CH}_{3} \mathrm{OH}\right)$, Ausb. $1.195 \mathrm{~g}(90.7 \%) .-\mathrm{C}_{15} \mathrm{H}_{21} \mathrm{NO}_{3}$ (263.3) Ber. C 68.4 H 8.04 N 5.3 Gef. C 68.5 H 7.98 N 5.4 Mol.-Masse 263 (ms). - IR: 1790; 1640; $1420 \mathrm{~cm}^{-1}$. - ${ }^{1} \mathrm{H}-\mathrm{NMR}\left(\mathrm{CDCl}_{3}, 250 \mathrm{MHz}\right): 1.00\left(\mathrm{~s}, 3 \mathrm{H}, \mathrm{CH}_{3}\right), 1.08\left(\mathrm{~s}, 3 \mathrm{H}, \mathrm{CH}_{3}\right), 1.15\left(\mathrm{~s}, 3 \mathrm{H}, \mathrm{CH}_{3}\right)$, $1.62-1.74(\mathrm{~m}, 1 \mathrm{H}), 1.78-1.96(\mathrm{~m}, 3 \mathrm{H}), 1.97-2.15(\mathrm{~m}, 3 \mathrm{H}), 2.36(\mathrm{ddd}, \mathrm{J}=4.5 / 10.8 / 13.6 \mathrm{~Hz}, 0.7 \times 1 \mathrm{H})$, 2.30-2.45 (m, $0.3 \times 1 \mathrm{H}), 3.59-3.78\left(\mathrm{~m}, 0.7 \times 2 \mathrm{H},-\mathrm{NCH}_{2}-\right.$ ). 3.80 (ddd, J = 3.8/7.3/12.8 Hz, $0.3 \times 1 \mathrm{H}$, $-\mathrm{NCH}_{2}{ }^{-}$), 3.94 (ddd, J = 3.8/8.1/12.8 Hz, $0.3 \times 1 \mathrm{H},-\mathrm{NCH}_{2}-$ ), $4.95(\mathrm{dt}, \mathrm{J}=3.9 / 8.5 \mathrm{~Hz}, 0.7 \times 1 \mathrm{H}$, $-\mathrm{NC}=\mathrm{CH}-), 5.20(\mathrm{dt}, \mathrm{J}=3.6 / 8.4 \mathrm{~Hz}, 0.3 \times 1 \mathrm{H},-\mathrm{NC}=\mathrm{CH}-), 7.07-7.13(\mathrm{~m}, 0.3 \times 1 \mathrm{H},-\mathrm{NCH}=\mathrm{C}-) .7 .16$ (dt, $\mathrm{J}=2.1 / 8.5 \mathrm{~Hz}, 0.7 \times 1 \mathrm{H},-\mathrm{NCH}=\mathrm{C}-$ ). - Rotamerenverhältnis $3 / 7$.

b) $13.17 \mathrm{~g} \mathrm{(50} \mathrm{mmol)} \mathrm{11,} 1.32 \mathrm{~g} \mathrm{Pd} / \mathrm{C}, 100 \mathrm{ml}$ THF, $3.5 \mathrm{~h}, 105^{\circ}$. Das Filtrat wurde i. Vak. eingeengt und der Rückstand aus Hexan/Ethylacetat umkristallisiert. Ausb. $10.78 \mathrm{~g}(81.9 \%)$.

(1,2,3,4-Tetrahydro-1-pyridyl)-I(IS, 3R)-1-hydroxy-3-hydroxymethyl-2,2,3-trimethylcyclopentyllketon (19)

$0.267 \mathrm{~g}(1 \mathrm{mmol}) 12,3 \mathrm{~h}$. - Das Filtrat wurde i. Vak. eingeengt und der Rückstand mit Ethylacetat versetzt. Es wurde mit lproz. $\mathrm{NaHSO}_{4}$-Lösung $(3 \times)$, mit $\mathrm{H}_{2} \mathrm{O}(3 \times)$ geschüttelt, getrocknet $\left(\mathrm{Na}_{2} \mathrm{SO}_{4}\right)$, eingeengt (i. Vak.) und aus Ethylacetat umkristallisiert. Farblose Kristalle, Schmp. $130-134^{\circ},|\alpha|_{546}=-16.4^{\circ}$, $[\alpha]_{578}=-11.9^{\circ}\left(\mathrm{c}=1.1, \mathrm{CH}_{3} \mathrm{OH}\right)$, Ausb. $0.218 \mathrm{~g}(81.7 \%) .-\mathrm{C}_{15} \mathrm{H}_{25} \mathrm{NO}_{3}$ (267.4) Ber. C 67.4 H 9.42 N 5.2 Gef. C 67.4 H 9.35 N 5.3 Mol.-Masse 267 (ms). - IR: 1610; $1020 \mathrm{~cm}^{-1}$. - ' ${ }^{1} \mathrm{H}-\mathrm{NMR}\left(\mathrm{CDCl}_{3}\right.$, $360 \mathrm{MHz}$ ): $0.83\left(\mathrm{~s}, 6 \mathrm{H}, \mathrm{CH}_{3}\right), 1.09\left(\mathrm{~s}, 3 \mathrm{H}, \mathrm{CH}_{3}\right), 1.58(\mathrm{ddd}, \mathrm{J}=4.3 / 10.8 / 13.3 \mathrm{~Hz}, 1 \mathrm{H}), 1.65-2.10(\mathrm{~m}$, $6 \mathrm{H}), 2.98$ (ddd, J $=8.6 / 10.7 / 14.3 \mathrm{~Hz}, 1 \mathrm{H}), 3.26\left(\mathrm{~d}, \mathrm{~J}=10.8 \mathrm{~Hz}, 1 \mathrm{H},-\mathrm{CH}_{2} \mathrm{O}-\right), 3.55(\mathrm{~d}, \mathrm{~J}=10,8 \mathrm{~Hz}, 1 \mathrm{H}$, $-\mathrm{CH}_{2} \mathrm{O}-$ ), 3.58 (m, n.a. = nicht aufgelöst, $1 \mathrm{H},-\mathrm{NCH}_{2}-$ ). 3.87 (m, n. a., $1 \mathrm{H},-\mathrm{CH}_{2} \mathrm{O}-$ ), 4.35 (s breit, $1 \mathrm{H}$, $\mathrm{D}_{2} \mathrm{O}$-Austausch, $\left.\mathrm{OH}\right), 4.83$ (m, n. a., $\left.0.8 \times 1 \mathrm{H},-\mathrm{NC}=\mathrm{CH}-\right), 5.13(\mathrm{~m}$, n. a., $0.2 \times 1 \mathrm{H},-\mathrm{NC}=\mathrm{CH}-), 6.11(\mathrm{~s}$ breit, $1 \mathrm{H}, \mathrm{D}_{2} \mathrm{O}$-A ustausch, $\mathrm{OH}$ ), 7.15 (m, n. a., $0.2 \times 1 \mathrm{H},-\mathrm{NCH}=\mathrm{C}$-), 7.73 (d breit, $0.8 \times 1 \mathrm{H},-\mathrm{NCH}=\mathrm{C}$-). - Rotamerenverhältnis $2 / 8$. 
(1,2,3,4-Tetrahydro-1-pyridyl)-I(IS, 3R)-1-hydroxy-3-methoxymethyl-2,2,3-trimethylcyclopentyl/keton (20)

$0.281 \mathrm{~g} \mathrm{(1} \mathrm{mmol)} \mathrm{13,} 3 \mathrm{~h}$. - Das Filtrat wurde i. Vak. eingeengt und der Rückstand zentrifugalchromatographisch gereinigt (Hexan/Ethylacetat $=60 / 40)$. Farblose Kristalle, Schmp. $48-50.5^{\circ},|\alpha|_{546}=-34.1^{\circ}$. $[\alpha]_{578}=-28.3^{\circ}\left(\mathrm{c}=1.02, \mathrm{CH}_{3} \mathrm{OH}\right)$, Ausb. $0.225 \mathrm{~g}(80.1 \%) .-\mathrm{C}_{16} \mathrm{H}_{27} \mathrm{NO}_{3}(281.4)$ Ber. C $68.3 \mathrm{H} 9.67$ N 5.0 Gef. C 68.2 H 9.55 N 5.0 Mol.-Masse 281 (ms). - IR: 3500-3250;1610; $1105 \mathrm{~cm}^{-1}$. - 'H-NMR $\left(\mathrm{CDCl}_{3}, 360 \mathrm{MHz}\right): 0.83$ (s, $\left.3 \mathrm{H}, \mathrm{CH}_{3}\right), 0.85\left(\mathrm{~s} .3 \mathrm{H}, \mathrm{CH}_{3}\right), 1.05\left(\mathrm{~s}, 3 \mathrm{H}, \mathrm{CH}_{3}\right), 1.59$ (ddd, J = 4.5/10.8/ $13.1 \mathrm{~Hz}, 1 \mathrm{H}$ ), $1.64-2.32(\mathrm{~m}, 6 \mathrm{H}), 3.02$ (ddd. J $=8.1 / 10.5 / 14.0 \mathrm{~Hz}, 1 \mathrm{H}), 3.03(\mathrm{~d}, \mathrm{~J}=9.4 \mathrm{~Hz}, 1 \mathrm{H}$, $-\mathrm{CH}_{2} \mathrm{O}$-), 3.30 (d, J = 9.4 Hz, $1 \mathrm{H},-\mathrm{CH}_{2} \mathrm{O}-$ ). 3.42 (s, $3 \mathrm{H},-\mathrm{OCH}_{3}$ ), 3.63 (ddd, J = 3.8/8.2/13 Hz, $0.8 \times 1 \mathrm{H}$, $-\mathrm{NCH}_{2}$-), 3.83 (ddd, J = 4.0/8.0/13 Hz, $0.8 \times 1 \mathrm{H}$. $-\mathrm{NCH}_{2}-$ ), 4.06-4.28(m, n. a., $\left.0.2 \times 2 \mathrm{H},-\mathrm{NCH}_{2}-\right), 4.80$ (dt, J = 4.3/8.5 Hz, $0.8 \times 1 \mathrm{H},-\mathrm{NC}=\mathrm{CH}-), 5.12\left(\mathrm{~m}, \mathrm{n}\right.$. a.. $0.2 \times 1 \mathrm{H},-\mathrm{NCH}=\mathrm{C}$-), $5.68\left(\mathrm{~s}, 1 \mathrm{H}, \mathrm{D}_{2} \mathrm{O}\right.$-Austausch, $\mathrm{OH}$ ), 7.21 (m, n. a., $0.2 \times 1 \mathrm{H},-\mathrm{NCH}=\mathrm{C}-$ ), $7.80 / \mathrm{d}, \mathrm{J}=8.5 \mathrm{~Hz}, 0.8 \times 1 \mathrm{H},-\mathrm{NCH}=\mathrm{C}-$ ), - Rotamerenverhältnis $2 / 8$.

\section{Literatur}

1 T. Shono, Y. Matsumura, K. Tsubata, Y. Sugihara. Tetrahedron Lett. 23, 1201 (1982); Übersichtsartikel: H. E. Zaugg, Synthesis 1984, 85; H. E. Zaugg. ibid. 1984, 181; G. R. Lenz, ibid. 1978, 489.

2 Vgl. hierzu: M. G. Van Campen, C. H. Tilford (Merrell Co.), Brit. Pat. 756686 (1956), C. A. 52. 3869 h (1958) und G. P. Claxton, F. G. Fallon (Merrell Inc.) US-Pat. 3306895 (1967); C. A. 68, 394865 (1968).

3 Sandoz Ltd., Neth. Appl. 6408293 (1965); C. A. 64, 2089 e (1966).

4 K. Nyberg, Synthesis 1976, 545 .

5 M. Mitzlaff, K. Warning und H. Jensen, Liebigs Ann. Chem. 1978, 1713.

$6 \mathrm{~K}$. Warning und M. Mitzlaff, Tetrahedron Lett. 1979, 1565 und dort zit. Lit.

7 J. K. Stille und Y. Becker, J. Org. Chem. 45.2139 (1980).

8 R. Willstätter und F. Königsberger, Ber. Dtsch. Chem. Ges. 56, 2107 (1923).

9 O. Aschan, Ber. Dtsch. Chem. Ges. 27, 649 (1894).

10 Wegen der basischen Reaktionsbedingungen wurde das Verfahren nicht auf (R)-7 angewandt.

11 R. F. Heck, Palladium Reagents in Organic Syntheses, S. 19, Academic Press, London 1985.

12 W. C. Still, M. Kahn und A. Mitra, J. Org. Chem. 43, 2923 (1978). 